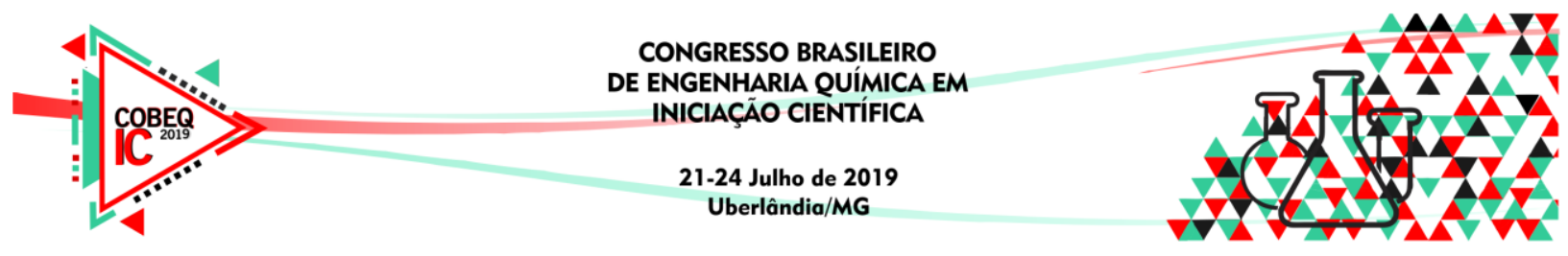

\title{
FILMES BIODEGRADÁVEIS FORMADOS POR TANINOS CONDENSADOS E ÁLCOOL POLIVINÍLICO MODIFICADO
}

\author{
N.P. CAMPIM e G.M.D. FERREIRA \\ Universidade Federal de Lavras, Departamento de Química \\ E-mail: natalia.campim1@estudante.ufla.br
}

\begin{abstract}
RESUMO - A busca pelo desenvolvimento social, aliando crescimento econômico e baixo impacto ambiental, fez com que vários setores industriais procurassem alternativas sustentáveis para a obtenção de produtos ambientalmente amigáveis em larga escala. Nesse sentido, produtos baseados em materiais biodegradáveis, como certos polímeros, têm recebido destaque, pois podem minimizar os impactos gerados no meio ambiente. Assim, analisando as características desejáveis de polímeros biodegradáveis e o vasto território vegetal brasileiro, que não tem sido aproveitado de maneira tão eficiente, esse trabalho investigou a formação de filmes biodegradáveis constituídos pelo polímero poli (vinil álcool) modificado, PVSiOH, e por taninos condensados oriundos de barbatimão, TCB. Os filmes foram obtidos pelo método de casting a partir de misturas de PVSiOH e TCB. A concentração dos compostos e o pH do meio na mistura geradora do filme foi investigada para avaliar a interação entre os componentes.
\end{abstract}

\section{INTRODUÇÃO}

Atualmente, os polímeros possuem diversas finalidades industriais e comerciais, sendo utilizados em muitas atividades cotidianas fundamentais à economia, saúde humana e animal, construção civil, vestuário, cosméticos, indústria automobilística e muitas outras aplicações que se expandem através de novas pesquisas e descobertas. Essa gama de aplicação deve-se à utilização de polímeros com diferentes estruturas que podem melhorar as propriedades dos materiais (CANEVAROLO, 2010). Dentre as possíveis formas de organização e apresentação dos polímeros em materiais, os filmes poliméricos, isto é, cadeias agrupadas de polímeros formando uma fina camada de material, em geral não porosa, (SUNTORNNOND et al., 2015) apresentam diversas aplicações. Por exemplo, na alimentação estão sendo criados para conservação de alimentos; na medicina, para revestimento de algumas partes do corpo, dentre várias outras.

A despeito da versatilidade e aplicabilidade de filmes poliméricos, há uma grande preocupação com relação aos problemas ambientais relacionados a estes tipos de materiais, abrindo destaque para os materiais poliméricos biodegradáveis, ou seja, polímeros nos quais a degradação resulta da ação de microrganismos de ocorrência natural como bactérias, fungos e algas, podendo ser consumidos em semanas ou meses sob condições favoráveis de biodegradação (MOHANTY et al., 2005). Um polímero biodegradável com grande capacidade de formar filmes é o poli(vinil álcool), PVA, um polímero que apresenta boas 


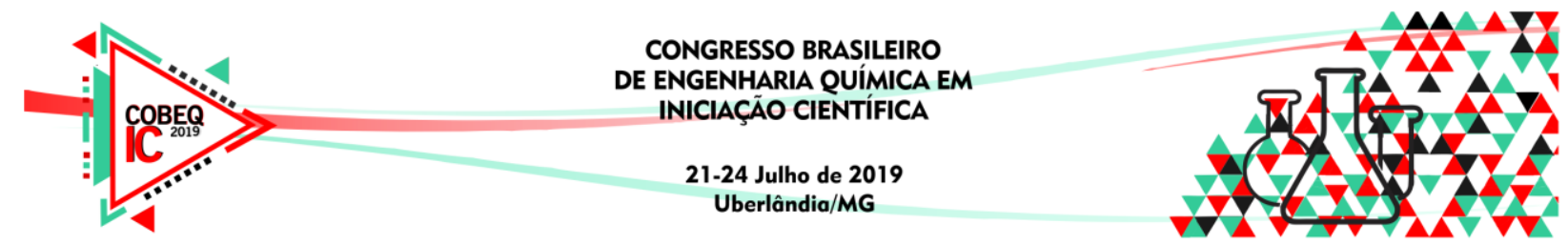

propriedades mecânicas, além de alta estabilidade. O interesse crescente da sociedade pelo uso de materiais "ecologicamente corretos" faz também com que os compostos de origem vegetal retomem o espaço ocupado pelos compostos sintéticos em vários setores. Um conjunto de polímeros biodegradáveis, ainda com pouco destaque, mas que podem ser muito utilizados como modificadores de propriedades de filmes poliméricos, são os taninos condensados (TC). Esses compostos fenólicos são definidos como um complexo heterogêneo de polifenóis de origem vegetal com alto peso molecular (500 a $3000 \mathrm{~g} \mathrm{~mol}^{-1}$ ) (FERREIRA e NOGUEIRA, 2000; AGANGA e MONASE, 2001) com eficiência considerável para formar ligações intermoleculares e intramoleculares em redes de polímeros.

Os taninos dividem-se em dois principais grupos: hidrolisáveis e condensados, embora MAKKAR (2003) ressalte a existência de taninos que são formados por uma mistura de ambos os grupos. Além disso, taninos condensados podem conter ácido gálico em sua cadeia, o que pode resultar em elevada atividade biológica do composto (SCHOFIELD, 2001). Pesquisas publicadas sobre TC juntamente com PVA para formação de filmes ainda são restritas na literatura, principalmente envolvendo PVA modificado. Assim, neste estudo, avaliou-se a interação do polímero PVA modificado com grupos silanóis com taninos condensados obtidos de barbatimão para avaliar e compreender o processo de formação dos filmes desses compostos.

\section{OBJETIVOS}

\subsection{Objetivo Geral}

Sintetizar e caracterizar filmes formados por álcool polivinílico modificado com grupo silanol (PVSiOH) e taninos condensados de barbatimão (TCB), estudando o comportamento de misturas desses componentes em meio aquoso.

\subsection{Objetivos Específicos}

2.2.1. Avaliar a interação entre PVSiOH e TCB em misturas aquosas, avaliando o efeito do $\mathrm{pH}$ e da concentração dos componentes nas misturas.

2.2.2. Obter filmes de blendas poliméricas formados por PVSiOH e TCB.

\section{MATERIAIS E METODOLOGIA}

O polímero PVSiOH foi gentilmente fornecido pela Kuraray e o tanino condensado foi obtido segundo metodologia descrita por (MORI et al., 2013) Água deionizada foi utilizada como solvente para o preparo de todas as soluções. Para os estudos de efeito do $\mathrm{pH}$, soluções aquosas estoques de PVSiOH e TCB, ambas com concentrações iguais a $0,200 \% \mathrm{~m} / \mathrm{v}$, foram preparadas e misturadas em diferentes tubos de vidro de $40 \mathrm{~mL}$. As misturas foram realizadas adicionando-se $8,00 \mathrm{~mL}$ de cada solução estoque de forma a obter concentrações finais de $0,100 \% \mathrm{~m} / \mathrm{v}$ de cada componente. Cada solução obtida teve seu $\mathrm{pH}$ ajustado utilizando soluções de $\mathrm{HCl}$ ou $\mathrm{NaOH}$ de concentrações iguais a 0,1 ou $1 \mathrm{molL}^{-1}$. Os valores de $\mathrm{pH}$ variaram entre 2,51 e 10,91. Para os estudos do efeito da concentração, soluções estoques de $\mathrm{PVSiOH}$ e TC, ambas com concentrações iguais a $0,200 \% \mathrm{~m} / \mathrm{v}$, foram preparadas e volumes 


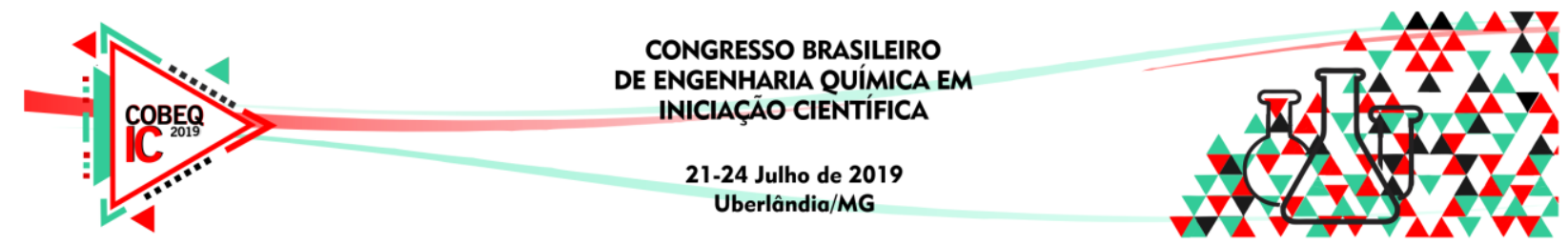

adequados foram adicionados em diferentes tubos de vidro de $40 \mathrm{~mL}$ a fim de obter misturas de PVSiOH de concentração $0,500 \% \mathrm{~m} / \mathrm{v}$, mas com concentrações variadas de TCB. As concentrações de TCB variaram entre 0 e $2,00 \% \mathrm{~m} / \mathrm{v}$. A turbidez de todos os sistemas obtidos após a mistura das soluções estoques, e que não levaram à formação de precipitado, foram realizadas com o auxílio de um turbidímetro MS tecnopon TB-2000. Algumas misturas de PVSiOH e TCB, adequadamente escolhidas em função das características dos sistemas, foram utilizadas para preparar filmes pelo método de casting (BORGES et al., 2017), utilizando placas de Teflon como suporte.

\section{RESULTADOS E DISCUSSÕES}

\subsection{Efeito do pH sobre a interação TCB + PVSiOH em solução aquosa}

A Figura 1 apresenta o efeito do $\mathrm{pH}$ sobre a turbidez de misturas formadas por TCB $(0,100 \% \mathrm{~m} / \mathrm{v})$ e PVSiOH $(0,100 \% \mathrm{~m} / \mathrm{v})$. Em valores de $\mathrm{pH}$ menores do que 2,51 ocorreu precipitação intensa na solução, impedindo a leitura de turbidez. $\mathrm{O} \mathrm{pH}$ do meio modifica a carga das espécies em solução (TCB e PVSiOH), levando a forças eletrostáticas intensas que podem favorecer a formação de agregados em solução. Em valores de pH mais elevados, os dois polímeros negativamente carregados impedem a formação desses complexos. Baseados nesses estudos, os demais experimentos foram realizados no $\mathrm{pH}$ inicial da mistura, ou seja, próximo de 6. Nessas condições, o complexo PVSiOH-TCB é solúvel.

Figura 1 - Turbidez em função do pH do meio para a mistura de TCB $(0,100 \% \mathrm{~m} / \mathrm{v}) \mathrm{e}$ $\mathrm{PVSiOH}(0,100 \% \mathrm{~m} / \mathrm{v})$.

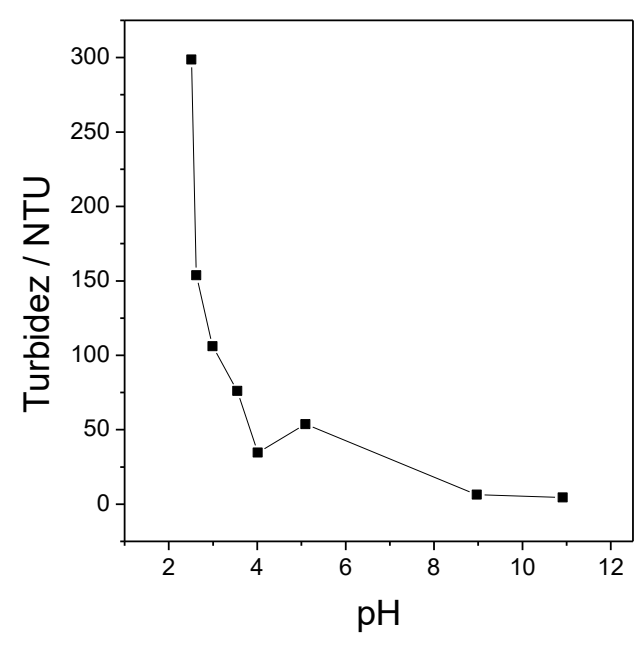

\subsection{Efeito da concentração sobre a interação TCB + PVSiOH}

Referente ao efeito da concentração das espécies de PVSiOH e TCB sobre a turbidez das misturas, as características visuais dos sistemas obtidos foram registradas. A Figura 2 mostra o efeito da concentração sobre a turbidez das misturas de PVSiOH + TCB em função da concentração de tanino avaliadas para os sistemas formados por concentração de PVSiOH igual a $0,500 \% \mathrm{~m} / \mathrm{v}$. 


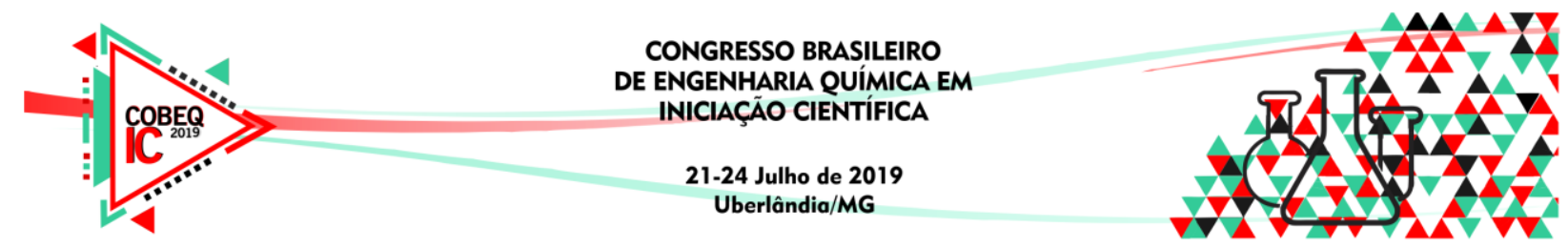

Figura 2 - Turbidez em função da concentração (em \% m/v) de TCB para misturas de $\mathrm{PAVSiOH}(0,500 \% \mathrm{~m} / \mathrm{v})+\mathrm{TCB}$.

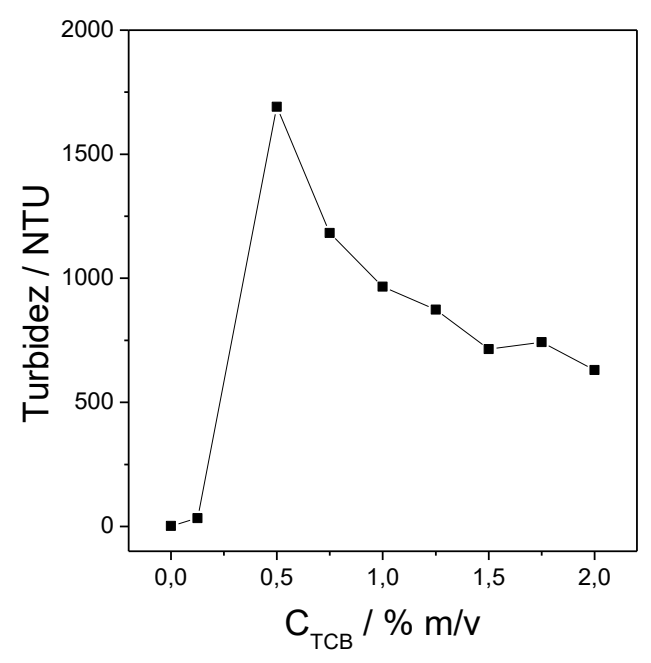

$\mathrm{O}$ aumento da concentração de TCB até valores de $0,125 \% \mathrm{~m} / \mathrm{v}$ afetou levemente a turbidez da mistura. Entretanto, quando a concentração aumentou para 0,500\% m/v, um aumento abrupto ocorreu na turbidez da solução, indicando a formação de agregados macroscópicos que permaneceram em suspensão. À medida que a concentração de TCB voltou a aumentar, a turbidez da solução diminuiu exponencialmente, sugerindo a solubilização parcial dos agregados formados. Em baixas concentração de TBC, a turbidez é baixa provavelmente devido à falta de concentração suficiente para que a interação TCBPVSiOH ocorra. Entretanto, com o aumento da concentração a partir de $0,125 \% \mathrm{~m} / \mathrm{v}$, o aumento da turbidez sugere o início da interação. Esse processo é similar a outros processos cooperativos, como o processo envolvendo formação de micelas de surfactante e a agregação entre polímeros e surfactantes, que ocorrem por meio de interações hidrofóbicas, ou seja, interações que ocorrem pelo aumento da entropia do sistema (FERREIRA et al., 2017). Baseados nos estudos de turbidez em função da concentração, outras concentrações de polímero precisam ser investigadas para avaliar o efeito de turbidez e precipitação, levando a uma melhor possibilidade de escolha da mistura para formação dos filmes.

\subsection{Formação dos filmes de PVSiOH-TCB}

Outras concentrações de polímero também foram avaliadas para diversas concentrações de TCB. Na realização deste teste, com as concentrações de 0,100, 0,500 e 1,000\% m/v, foi possível observar que a leitura da turbidez se tornou cada vez mais difícil, uma vez que, como a leitura foi simultânea ao preparo da soluções de tanino-PVSiOH, havia aglomerados presentes na solução que não precipitaram imediatamente.

Com algumas misturas de tanino-PVSiOH $(0,100 \% \mathrm{~m} / \mathrm{v})$, foram preparados filmes em placas de Teflon (Figura 3). A Figura 3a mostra os filmes preparados com algumas dessas misturas antes de serem removidos, enquanto a Figura $3 b$ mostra as características desses filmes observadas durante a remoção deles. É possível observar que os filmes ficaram quebradiços, sendo que, da esquerda para a direita, o aumento da concentração de TCB reduziu a plasticidade do material. 


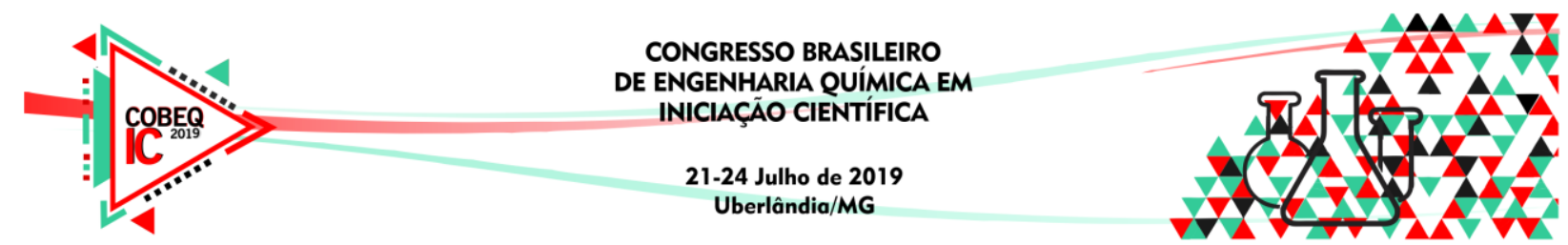

Figura 3 - Filmes com soluções tanino-PVSiOH 0,100\% m/v.

(a)

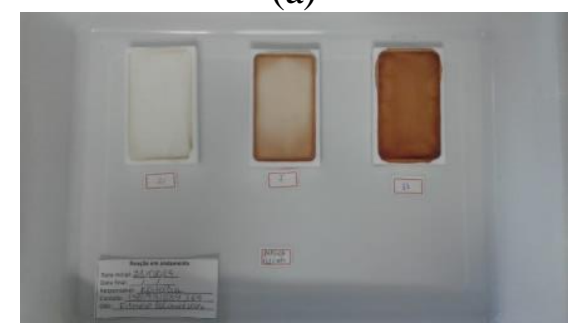

(b)

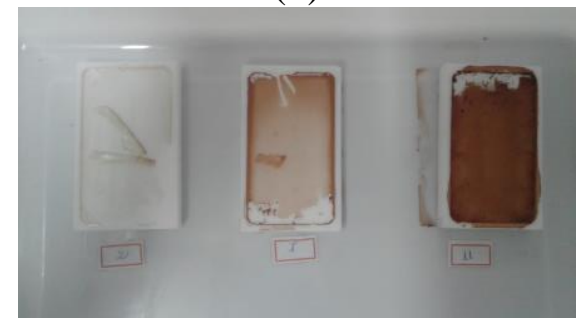

Para melhorar a plasticidade do material, concentração mais elevadas de PVSiOH foram avaliadas. A Figura 4 mostra as características dos filmes, durante a remoção, obtidas a partir de algumas misturas de tanino-PVSiOH $0,500 \% \mathrm{~m} / \mathrm{v}$.

Figura 4 - Remoção dos filmes com soluções tanino-PVSiOH 0,500\% m/v.

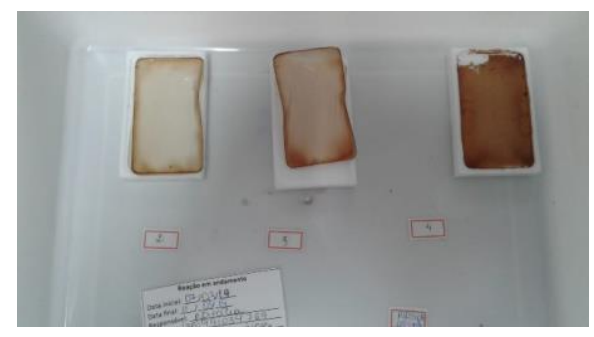

As seguintes características foram observadas para os filmes na concentração de $\mathrm{PVSiOH} 0,500 \% \mathrm{~m} / \mathrm{v}$ : primeira placa: filme removido com facilidade; segunda placa: filme removido com facilidade; terceira placa: filme quebradiço. Mediante as observações feitas com algumas soluções de tanino-PVSiOH, $0,100 \% \mathrm{~m} / \mathrm{v}$ e $0,500 \% \mathrm{~m} / \mathrm{v}$, percebe-se que o aumento de concentração do TCB torna os filmes mais quebradiços. Elevadas concentrações de TCB favorecem formações de interações intermoleculares TCB-TCB, diminuindo a resistência dos filmes formados. Com as misturas de TCB-PVSiOH 1,00\% m/v não foram preparados nenhum filme polimérico, devido ao fato de todas a soluções terem formação de precipitado.

\section{CONCLUSÕES}

Mediante o principal objetivo do trabalho, foi possível observar que houve interação entre o tanino condensado e álcool polivinílico modificado em solução aquosa, sendo esta interação dependente do $\mathrm{pH}$ da mistura e da concentração de TCB. A resistência dos filmes formados dependeu da concentração de TCB e PVSiOH, sendo que o aumento da concentração do TCB levou a formação de filmes mais quebradiços.

\section{REFERÊNCIAS}




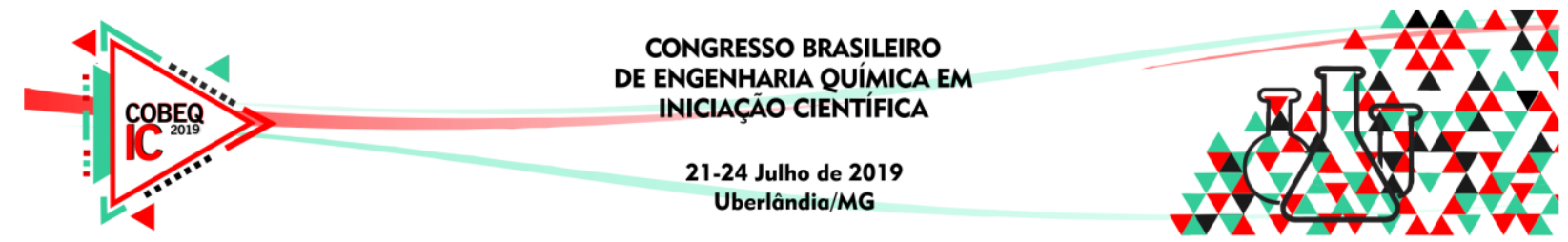

AGANGA, A.A.; MONASE, K.W. Tannin content, nutritive value and dry matter digestibility of Lonchocarpus capasa Zizyphus mucronata Sclerocarya birrea Kirkia acuminata and seeds. Animal Feed Science and Technology v.91, p.107-113, 2001.

BORGES, A. L. G; LAURINDO, J. B.; PREPARAÇÃO DE FILMES BIODEGRADÁVEIS DE AMIDO DE MANDIOCA PELOS", MÉTODO TAPE-CASTING E COM SECAGEM AO SOL", p. 2237-2249. In: Anais do Xll Congresso Brasileiro de Engenharia Química em Iniciação Científica [= Blucher Chemical Engineering Proceeding, v1, n.4]. ISSN Impresso:2446-8711. São Paulo: Blucher, 2017.

CANEVAROLO, Jr Sebastião V. Ciência dos polímeros: Um texto básico para tecnólogos e engenheiros. $3^{\mathrm{a}}$ edição, 2010

FERREIRA, E.C.; NOGUEIRA, A.R. Vanillin-condensed tannin study using flow injection spectrophotometry. Talanta v.51, p.1-6, 2000.

FERREIRA, G.M.D; FERREIRA, G.M.D; HESPANHOL, M.C.; AGUDELO, A.J.P.; REZENDE, J.P.; PIRES, A.C.S.; SILVA, L.S H.M. Effect of Acetonitrile and N, NDimethylformamide on the Formation of Poly(ethylene oxide)-Sodium Alkyl Sulfate Aggregates. Journal of the Brazilian Chemical Society v.29, p.45-57, 2017.

MAKKAR, H.P.S. Effect and fate of tannins in ruminant animals, adaptation to tannins, a1nd strategies to overcome detrimental effects of feeding tannin-rich feeds. Small Ruminant Research v.49, p.241-256, 2003.

MOHANTY, A.K.; MISRA, M.; DRZAL, L. T.; SELKE, S.E.; HARTE, B.R.; HINRICHSEN, G. Natural Fibers, Biopolymers, and Biocomposites: An Introduction. In: Mohanty, A. K.; Misra, M.; Drzal, L. T. Natural Fibers, Biopolymers, and Biocomposites. Boca Raton: Taylor \& Francis, 2005.

MORI, C.L.S; PASSOS, N.A.; OLIVEIRA, J.E.; MATTOSO, H.C.; MORI, F.A.; CARVALHO, A.G.; FONSECA, A.S.; TONOLI, G.H.D. Electrospinning of zein/tannin bio-nanofibers, Industrial Crops and Products v.52, p.298-304, 2013.

Norma ASTM D6400-04. Standard Specification for Compostable Plastics.

SCHOFIELD, P.; MBUGUA, D.M.; PELL, A.N. Analysis of condensed tannins: a review. Animal Feed Science and Technology v.91, p.21-40, 2001.

SUNTORNNOND, R.; AN, J.; YEONG, W.Y.; CHUA, C.K. Biodegradable Polymeric Films and Membranes Processing and Forming for Tissue Engineering. Journal Macromolecular Materials and Engineering: a review v.9, p.858-861, 2015. 